\title{
Using Focus Groups to Listen, Learn, and Lead in Higher Education
}

Written by: Mona J. E. Danner, J. Worth Pickering, and Tisha M. Paredes Published by: Sterling, VA: Stylus Publishing. 192 pages.

Reviewed by: Tory E. Dellafiora, Senior Assistant Director of Outreach and Assessment, the Career Center, Florida State University

Using Focus Groups to Listen, Learn, and Lead in Higher Education is an excellent addition to the bookshelf of anyone seeking to expand their research and assessment toolbelt. Mona J. E. Danner, J. Worth Pickering, and Tisha M. Paredes serve as knowledgeable guides on the process of conducting focus groups, a methodology that may intimidate those unfamiliar with their use on college campuses. The book is a quick read, but the thorough treatment of the topic leaves one feeling equipped to use this method in their own research and assessment processes.

For context, all three authors have worked with the Old Dominion University (ODU) team that has conducted more than 100 focus groups over the last 30 years. Danner is a professor and the chair of the Department of Sociology and Criminal Justice, Pickering serves as a consultant in the Office of Institutional Effectiveness and Assessment, and Paredes is assistant vice president for institutional effectiveness and assessment. In addition to their work at ODU, they have facilitated training and workshops for several higher education associations and institutions. These professionals are well-versed in using focus groups in higher education, and their expertise and clarity shine throughout the volume.

The primary argument of the text is that focus groups are valid and valuable tools for gathering stakeholder input in higher education institutions. The authors offer a strong rationale for formalizing focus groups methods to create rigorous and relevant assessments that decision-makers can use effectively. They emphasize the steps taken 
(though not necessarily linearly) to streamline and standardize the development of focus groups studies. Most scholars, practitioners, and administrators will find the highly digestible approach helpful.

The book contains a foreword, preface, and nine chapters related to methodological considerations and detailed explanations of the ODU method. In addition, a substantial resource section contains 15 appendices. Jillian Kinzie of Indiana University-Bloomington's Center for Postsecondary Research and the National Survey of Student Engagement (NSSE) contributed the foreword. She frames the importance of listening to student voices and why qualitative methods, especially focus groups, make critical contributions to a complete assessment picture. In the preface, the authors outline the book's intent as a comprehensive volume for higher education professionals across functional areas and institutional roles. They also use this section to discuss how the ODU method has been honed and used over the years.

Though methodologically dense, the book provides the scaffolding necessary to defend and adequately frame the use of focus groups in research and assessment. The first chapter provides the primary research basis and backing for using focus groups as a research method. The authors introduce a phrase used often throughout the book, "rigorous and relevant." Chapter 2 introduces the 6-step ODU method around which the rest of the text is organized. The steps include the following:

1. determining whether focus group methodology will answer the research questions,

2. selecting focus group participants,

3. designing the moderator's guide,

4. selecting and training focus group moderators,

5. conducting focus groups, and

6. analyzing focus group data and reporting the findings.

In this chapter, the authors also highlight the subjects and topics for three vignettes used throughout the book and several documents included in the appendices.

Chapters 3 through 8 offer an in-depth exploration of each step of the ODU method. Each chapter includes critical information for constructing and running a valid assessment, focusing on the knowledge and logistics 
needed to achieve the goals of each step. The authors draw examples from the scenarios introduced in Chapter 2 to help readers apply focus group methodology in different contexts. Chapter 9 identifies things that can potentially go wrong in focus groups and how to create backup plans to preserve the research study. Finally, the book includes more than 50 pages of templates and examples in the appendices.

The primary strength of this volume lies in its clarity and process-driven approach to using focus groups. The book provides an excellent structure for conducting focus groups while also providing a clear theoretical framing to support this methodological choice. In addition, the authors clarify that though the text presents a step-by-step format, the actual process of preparing a focus group study does not always happen in such a linear fashion. The book also proves to be an incredible resource, with nearly one third of its 177 pages devoted to templates, checklists, and examples. As a result, readers will find everything they need to conduct a thorough focus group research study.

Finally, the book's principles have broad applicability in higher education. For example, in the introduction, the authors describe program evaluations, SWOT analyses, and institutional mission clarification as examples of when the ODU method for focus groups has been used successfully. One thing that will be especially useful for OTR professionals is a vignette focused on retention. We often want to know why someone left our institution, yet finding these students and getting them to participate in a focus group once they have left is extremely difficult, if not impossible. In the vignette, the authors reframe the idea for the retention study from determining why students leave to understanding what makes for a successful transition and encourages students to stay. When designing focus group studies, we must attend to the types of questions that this methodology can answer.

The biggest drawback of focus group methodology as defined by these authors is its heavy reliance on human resources. For example, the authors emphasize the importance of using external facilitators to moderate focus groups. While people connected with a department or program can develop facilitator guides and research questions for internal studies, they ultimately cannot moderate focus groups sessions. Further, the authors argue that facilitators can and should be compensated for their time, creating an additional burden on individuals interested in pursuing focus group research. One solution the authors 
offer is training multiple departments in focus group methodology and trading external facilitator responsibilities as research projects come up.

However, this approach assumes an investment in using focus groups to conduct qualitative research across multiple departments or units. At an institution like ODU, where a team has been deployed for several decades to coordinate this type of research with buy-in from the top down, it is easy to suggest that departments find colleagues willing to trade studies with them. However, finding partners may be more challenging for a single individual or department wanting to conduct this type of research. This omission feels like a significant gap in a text filled with otherwise concrete and clear instructions for the rest of the research process. A discussion of how to build an institutional culture invested in focus group methodology would be beneficial.

This volume is an excellent and valuable read for anyone involved in student orientation, transition, and retention. So often, as professionals, we lament the information we would collect "if only," but we are unable or unwilling to move beyond the surveys that form the core of most assessments. However, using focus groups allows for a more person-centered approach to gathering information without the time and human resources required for individual interviews. Particularly for a department or unit with a long history of anecdotal support for programs or services but little data to back up those claims, focus groups may provide a targeted and streamlined approach to gathering that evidence. Using the steps outlined in the text allows for a more rigorous research and assessment process beyond, "Well, I heard from this student...."

Books about research methods are often heavy, dense, and cumbersome, but Using Focus Groups to Listen, Learn, and Lead in Higher Education was a quick and engaging read with clear and actionable steps. The book has become a worthwhile addition to my own methods bookshelf. For those looking to add qualitative methods to their research and assessment portfolio, focus groups are a great start-and this volume is an excellent introduction. 